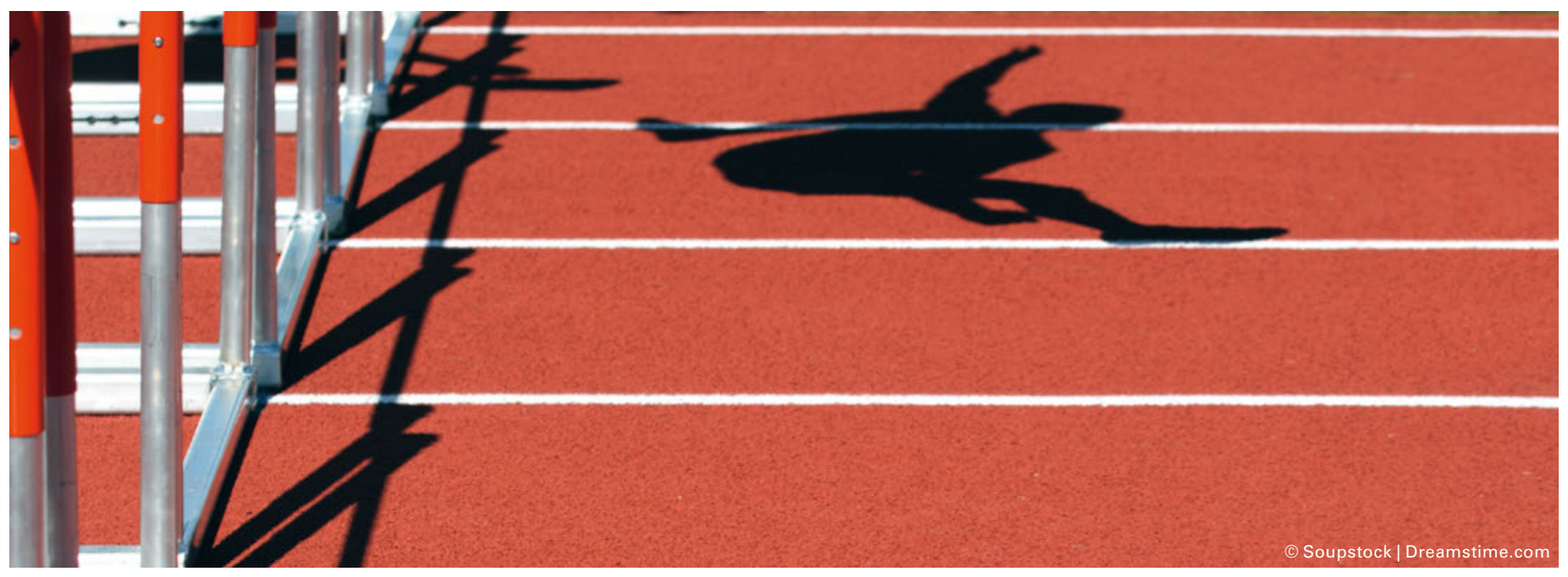

\title{
"Comment surmonter ses réticences et autres obstacles»
}

\author{
Marco Zalunardo ${ }^{a}$, Sebastian Krayer ${ }^{b}, B$ Bernhard Walderc, Thomas Brunner ${ }^{c}$, Peter Bauerfeind ${ }^{d}$, \\ Stefan Hartmeier ${ }^{d}$, Daniel Franzen ${ }^{e}$, Ludwig Jacobf, Peter Ammanng, Daniel Weilenmann ${ }^{g}$ \\ ${ }^{a}$ président de la SSAR; ${ }^{b}$ membre du comité de la SSAR; ${ }^{c}$ délégué de la SSAR; ${ }^{\text {d }}$ membre du comité de la SSG; ${ }^{e}$ délégué de la SSP; \\ ${ }^{\dagger}$ membre du comité de la SSVIR; ${ }^{9}$ délégué de la SSC
}

Des gastro-entérologues ont publié en 2011 dans le Swiss Medical Forum un article optimiste intitulé «La sédation au propofol en endoscopie gastro-intestinale - Une histoire à succès suisse» [1]. Le sujet de l'article, qui reflétait la pratique quotidienne en gastro-entérologie, avait déjà suscité auparavant de vifs débats à l'échelle européenne, des débats qui ont abouti à ce que certains anesthésistes et leurs sociétés nationales nient complètement aux gastro-entérologues la compétence à administrer le propofol [2]. Aucun consensus n'a pas pu être atteint à l'échelle européenne. Une réaction correspondante ne se fit pas attendre en Suisse. Certains anesthésistes ont, dans une lettre aux éditeurs, réagi de façon similaire à l'article susmentionné à l'encontre des gastro-entérologues [3]. La petite guerre était donc déclarée au sein de la profession et les positions prises. Une coopération fondamentale de l'anesthésie et de la gastro-entérologie dans ce domaine semblait à l'époque difficile à beaucoup d'endroits et même impossible au niveau de la profession.

C'est grâce à l'initiative et à la force de persuasion de différents membres des comités des deux sociétés de discipline que les comités professionnels de la SSAR et de la SSG se sont réunis pour débattre sur ce sujet malgré des circonstances défavorables. Ils se sont rencontrés pour la première fois en août 2012. L'atmosphère était tendue, les deux côtés s'attendant à une résistance ou même à une escalade. Rien de tel ne s'est produit. Une discussion ouverte avec des concessions des deux côtés: la condition d'une collaboration fructueuse. Chacun semblait disposé à surmonter ses réticences. Cette réunion a donné le coup d'envoi d'une coopération continue et fructueuse.

\section{L'objectif premier du groupe de travail} constitué à partir de la SSG et de la SSAR était la sécurité du patient.

L'objectif premier du groupe de travail constitué à partir de la SSG et de la SSAR était la sécurité du patient. Un papier de consensus avec des recommandations et des directives sur la sédation au propofol par les gastroentérologues a finalement pu être rédigé à l'issue de multiples séances. Le papier a, en dépit de la résistance, été approuvé et adopté comme directive gastro-entérologique 2013 à l'assemblée générale de la SSG. La responsabilité, les conséquences juridiques et le bien-être 
et la sécurité du patient ont en définitive été déterminants pour son adoption par les membres de la SSG présents.

L'idée avait été lancée de ne pas se limiter seulement àla gastro-entérologie et au propofol mais de définir des directives et des normes de sécurité valables en général qui étaient applicables indépendamment du spécialiste interventionnel et de la substance utilisée. Le groupe de travail a donc été élargi et ce, d'une part avec des anesthésistes spécialisés qui apportaient une grande expérience dans les analgo-sédations pour les interventions, et d'autre part avec d'autres sociétés de discipline effectuant régulièrement et fréquemment des analgo-sédations: cardiologie, radiologie interventionnelle et pneumologie. Le groupe de travail élargi a démarré en octobre 2014 sous l'égide de la SSAR. Toutes les sociétés de discipline participantes ont envoyé des délégués officiels. Un climat constructif a d'emblée régné dans ce groupe; peut-être aussi parce que certains délégués pouvaient déjà se prévaloir de longues années de collaboration multidisciplinaire fructueuse. D'importants thèmes ont été abordés, comme par exemple l'évaluation préalable des patients et de leurs risques, la profondeur de sédation "permise» ainsi que la période de jeûne jusqu’à l'intervention / la sédation. Une sédation modérée (stades I et II) ne pose pas encore d'exigences particulières au spécialiste interventionnel du point de vue anesthé-

\section{L'idée avait été lancée de ne pas se limiter seulement à la gastro-entérologie et au propo- fol mais de définir des directives et des normes de sécurité valables en général.}

siologique. Dans une sédation profonde (stades III et IV), le patient perd graduellement connaissance. L'altération de la respiration spontanée, des réflexes de défense et de la stabilité hémodynamique qui en résulte exige certaines aptitudes à identifier et traiter une insuffisance respiratoire et une situation circulatoire. En pratique clinique, l'introduction d'un gastroscope à l'état éveillé (stades de sédation I et II) est souvent mal tolérée par les patients et n'est plus guère acceptée. Les exigences des patients ont fortement augmenté ces dernières années, c'est pourquoi une sédation plus profonde temporaire n'est pas seulement souhaitable pour beaucoup d'interventions, en particulier cardiologiques, mais fait aussi partie intégrante de la pratique quotidienne depuis des années.
Le groupe de travail en a intensivement discuté. Le résultat de la discussion a été reproduit sous forme de papier de consensus et finalement accepté par toutes les sociétés de discipline, même par l'assemblée générale de la SSAR, où l'on pouvait sentir un vent contraire. On a assisté dans l'immédiat et à l'assemblée générale à des attaques et à des activités inédites pour la SSAR. La peur de perdre de l'influence, du contrôle, de la sécurité, de la qualité et naturellement aussi des sources de revenus primait. La responsabilité et d'éventuelles conséquences juridiques étaient également à l'ordre du jour. Une majorité des membres présents a cependant pu être convaincue de reconnaître les signes du temps et de soutenir cette collaboration multidisciplinaire.

\section{L'aboutissement dépend dans une proportion non négligeable des individus impliqués.}

Ce document, qui est publié dans le Swiss Medical Forum parallèlement à cet article, est la première démarche commune. [4] Des révisions et des adaptations seront nécessaires pour continuer à répondre aux besoins cliniques changeants. La prochaine étape est la mise en œuvre pratique dans les différentes sociétés de discipline. La SSAR soutiendra et accompagnera activement ici les autres disciplines en vue de l'instruction et de la formation continue durables de leurs membres sur la base du papier de consensus.

Cette «histoire à succès» montre une fois de plus que la conviction, la ténacité et surtout la volonté de consensus peuvent surmonter des obstacles apparemment insurmontables. L'aboutissement dépend dans une proportion non négligeable des individus impliqués. Si l'on met en avant des intérêts propres et le désir de se profiler aux dépens de la cause ou, plus précisément, du patient, il est alors improbable que l'on trouve un consensus.

\section{Références}

1 Inauen W, Külling D, Heuss LT, Bauerfeind P. La sédation au propofol en endoscopie gastro-intestinale - Une histoire à succès suisse. Forum Médical Suisse. 2011;11(51-52):961-2.

2 Perel A. Non-anaesthesiologists should not be allowed to administer propofol for procedural sedation: a Consensus Statement of 21 European National Societies of Anaesthesia. Eur J Anaesthesiol. 2011;28(8):580-4.

3 Knüsel R, Meister B, Schnider T. Propofolsedation in der gastrointestinalen Endoskopie: eine gefährliche Erfolgsgeschichte. Forum Médical Suisse 2012;12(25):519-21.

4 Zalunardo M, Krayer S, Brunner T, Walder B, Bauerfeind P, Hartmeier S, Ammann P, Weilenmann D, Jacob AL, Franzen D. Document de consensus de la SSAR, la SSG, la SSC, la SSCIR et la SSP: Recommandations et standards pour l'analgo-sédation administrée par un non-anesthésiste. Forum Med Suisse. 2016;16(45):969-72. 